the patient, for, contrary to what is generally supposed, the pain caused by cutting the grafts is quite trifling provided the instrument used is very sharp. This method has, however, its disadvantages and these chiefly concern the resulting scar in which subsequent contraction is only partially prevented, the surface is hard and closely adherent to underlying parts, whilst the epithelial covering is thin and easily breaks down into ulcers on irritation.

The method of skin-grafting which $I$ have practised on several occasions where the prevention of contraction was important and which I have found to give most satisfactory results is a combination of two of these methods, and consists in raising from some convenient part of the body a piece of the whole thickness of the skin surrounded by a narrow fringe of epithelium only, as in Thiersch's method. After determining the size and shape of the piece of skin to be transplanted in its whole thickness and marking it out if necessary, small superficial grafts (a) are cut on all sides of it with a very sharp razor. These are left attached at their bases to the margins of the main part of the graft and can be reflected on to it. The latter (A) is then dissected up in its whole thickness and all fat having been removed from its under surface with curved scissors or a very

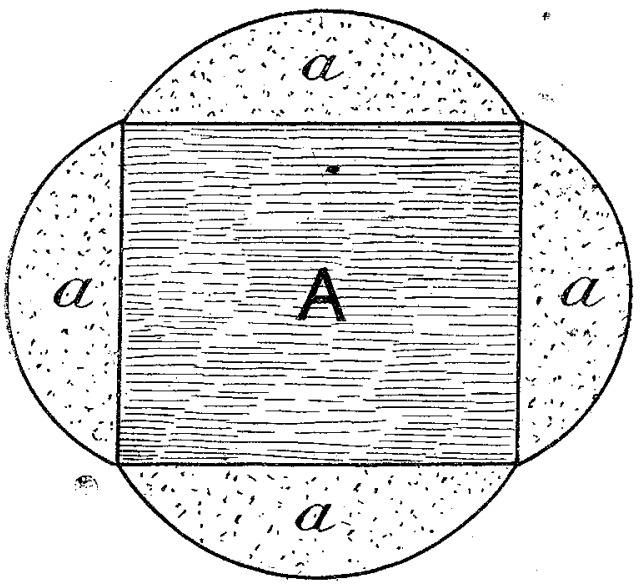

Diagram to represent a graft consisting of (A) a square piece of the whole thickness of the skin surrounded by $(a, a, a, a)$ a fringe of epithelium only.

sharp scalpel the whole graft is laid on the raw surface and the edges spread out with care and exactness; these rapidly adhere and by fixing the main part of the graft assist in ensuring its growth. The number of such grafts used depends, of course, on the shape and size of the surface to be covered. When the requisite number have been planted and the fringes exactly spread out they can be treated in the ordinary way as Thiersch grafts. The superficial epithelium from the central part is shed after a few days, as in all cases where the whole thickness of the skin has been transplanted, but the deeper parts remain and the wound is quickly covered over in a very satisfactory manner, the scar showing little tendency to contract and being less unsightly and less adherent to the underlying parts than that after grafting by the Thiersch method alone.

The wound caused by the removal of the graft can be treated in various ways. It may be possible to draw the edges together by sutures, a Thiersch graft may be put on that part from which the whole thickness of the skin has been removed, or it may be left to granulate. Care should of course be taken in grafting by this method that the graft be not taken from a hairy part of the body if the presence of hair in the new situation is at all objectionable, for the follicles are transplanted with the skin and the hair continues to grow. In this, as in all methods of skin-grafting where epithelial grafts are wholly or partially employed, attention to a few details is of great importance. The edges of the grafts must be accurately spread out. Much depends on the adhesion of the extreme edge and a little curling in or overlapping of a part already covered with epithelium may prevent its growth; any air bubbles or blood which may be under the graft must be removed by gentle pressure, this being employed, of course, by the use of some instrument or material which will not adhere to them; the preliminary treatment, too, of the surfaces from and to which the grafts are removed requires attention. It has been shown by experiments ${ }^{2}$ that although the vitality of epithelial grafts is not

1 Wentscher : Centralblatt für Chirurgie, Jan. 8th, 1898. impaired by keeping for a considerable time, or by moderate degrees of heat and cold, they are peculiarly susceptible to the action of chemical antiseptics; these surfaces, then, after having been rendered aseptic, should be treated for at least 24 hours before operation with some aseptic dressing such as sterilised gauze or lint wrung out of plain boiled water, similar precautions being taken with regard to the instruments used at the operation and the dressings applied at the time, so that no chemical antiseptic may come in contact with the grafts until they have become adherent in their new position.

Queen Anne-street, W.

\section{HYDATID OF THE LIVER; SUPPURATION, BURSTING INTO A BRONCHIAL TUBE, DRAINED, AND THE CYST WALL REMOVED THROUGH INCISION \\ INTO THE LUNG; RE- COVERY.}

BY JOHN JONES, M.D., B.S. LOND.

THE patient, a girl, aged eight years, was brought to me by her mother in July, 1895, on account of a "lump on the child's stomach." On examination there was found a smooth, elastic, rounded tumour lying in the right epigastric region, partly under cover of the lower sternal ribs of that side and extending downwards to within three and a half inches of the umbilicus. The ribs were bulged considerably outwards. Above the tumour was found to become continuous with the liver. There were no pain or tenderness over the tumour, no hydatid vibration, no jaundice, no ascites, and no albuminuria. The spleen was not enlarged, the heart-sounds were normal, and the apex beat was in its normal position. There was no pyrexia. The child's general health was said to be good, but she complained of "shortness of breath" and of "feeling tight across the stomach," especially after meals. The mother stated that she had noticed the "lump coming on" for nearly two years, but that it had not perceptibly increased in size during the past six months-not, in fact, since some of the fluid contents of the tumour had been removed by another practitioner for diagnostic purposes.

Aspiration of the cyst was suggested, but the mother would not consent to any surgical interference. From this time on the child was kept under observation. She remained in statu quo until the end of September, 1895, when she developed a dry cough. The dyspncea became more marked, the tumour became painful and tender, and the temperature, which had hitherto been normal, was constantly raised, but there were no definite rigors. On Oct. 4th the patient was seized with acute pain in the right infra-mammary region accompanied by vomiting. On examination the upper margin of the liver dulness, which had hitherto been at the upper border of the fifth rib, couid not be defined, the dulness being now found to extend above the nipple to the extent of one and a half inches. There were well-marked signs of consolidation of the lung in front and a pleuritic rub over the infra-mammary region of the affected side. On the 6th the patient had a severe rigor, followed by profuse perspiration. The temperature was $105.4^{\circ} \mathrm{F}$. The vomiting still persisted, and apparently it was independent of the ingestion of food. The cough was worse and small quantities of rusty sputum were expectorated. On the 7th she had two rigors. On the 8 th she had another rigor and diarrhoea set in. The cough was now very persistent and painful but the vomiting was much less. From now until the 18th she had one or more rigors daily, followed by profuse perspiration. The diarrhcea still persisted. There was slight jaundice of the conjunctivæ. 'l'he patient was rapidly losing ground. Enlarged veins became visible over the front of the lower part of the right chest. Immediately below and internal to the right nipple some cedema of the skin appeared, which gradually extended over the whole of the front of the right chest. Very loud moist sounds could be heard over the lower part of the front of the right chest. The tumour was still very painful and tense. On the 21st I was hurriedly sent for as the child was "choking." On arrival I found the patient 
propped up in an armchair and in a very collapsed state. It appeared that she had had a prolonged bout of coughing at the end of which she expectorated about an ounce of sputum of the colour of prune-juice and of a most offensive odour. In the course of a few minutes she had another paroxysm of coughing. A few drachms of fotid sputum only were expectorated with the first expulsive efforts. The act of coughing, which closely resembled that of whooping-cough but without the terminal whoop, persisted for fully a minute longer, ceasing only when the child fainted.

The mother now pleaded for an operation, although the bad prospects of recovery at this stage were fully explained to her. On examining the tumour it was found to be much less tense than it had been two days previously. There were no signs indicating adherence of the tumour to the abdominal parietes. It was inferred that the cedema of the skin covering the chest indicated that the abscess had burst into the lung at no great depth from the surface or else that it must have burst primarily into the pleural cavity and secondarily into the lung. It was therefore concluded that less immediate danger would ensue from an incision into the chest than from an incision through the abdominal wall. It was decided to make an incision at the spot where the cedema first appeared-i.e., immediately below and internal to the nipple. An anæsthetic was quite out of the question. The skin at the selected spot, after cleansing, \&c, was drawn up over the rib next above and a slightly crescentic incision one and $a$ half inches long was made through the whole thickness of the skin. The intercostals were detached from the upper margin of the fifth rib for a similar extent and a sinus forceps was then thrust through the pleura into the lung tissue for the distance of one inch from the external surface of the rib. On separating the blades some fotid pus exuded. Leaving the forceps in situ a scalpel was introduced between the blades and the pleura and lung tissue were divided horizontally to the extent of the skin incision. This was followed by a slight gush of blood and by several ounces of foetid pus. The forefinger was inserted into the wound and was found to enter into a cavity of about the size of a walnut, from the inferior part of which a track descended directly downwards which easily admitted the tip of the forefinger. A half-inch drainage-tube was inserted and a gauze-and-wool dressing was applied. In two hours' time the dressings were perfectly sodden with the discharge and on removing them the drainage-tube was found to have been expelled. The ratient had not expectorated any pus since the incision was made. The drainage-tube was re-inserted, but before fresh dressing could be applied a paroxysm of coughing set in which promptly expelled the tube. The flap of skin was then seen to be sucked in with each inspiration, thus effectually excluding the air yet permitting a free expulsion of pus. The drainage-tube was discarded, only an external dressing being applied. The paroxysms of cough, though now unattended with expectoration and of less frequent occurence, were not changed in character. Consequently some hours later, the patient having rallied somewhat, the wound was dilated and swabbed dry and a reflected light was thrown in. Nothing distinctive could be made out until a paroxysm of coughing occurred which caused a gush of discharge. After swabbing out the pus a pearly white substance could be seen at the bottom of the wound. This white substance was not visible when the paroxysm ceased. Caking this substance to be part of the cyst wall it was decided to wait and during the next paroxysm to seize the cyst wall in the blades of a forceps. During the paroxysm the cyst wall re-appeared in the lower part of the wound and was promptly seized by means of a Wells forceps and slight traction was made upon it. As more of the cyst wall became visible a second pair of forceps was applied behind the first. Traction made during the inter-paroxysmal periods was found to cause tearing of the cyst wall, consequently it was only applied during the paroxysms. The points of fixation of the forceps were alternately changed as more and more of the cyst wall appeared at the bottom of the wound. After 15 minutes the whole of the cyst wall was extracted almost intact. During its extraction about four ounces of the foetid pus was discharged, to the weight of which must be attributed the proneness of the cyst wall to tear, for on further examination of the latter its friability was not nearly so great as was at first suspected. In volume the size of the cyst wall was rather larger than a Jaffa orange. A little iodoform emulsion was syringed into the cavity and a 'gauze-and-wool dressing was applied externally. The character of the cough changed entirely after the extraction of the cyst wall, the pertussislike paroxysms disappearing there and then.

The after-history of the case was uneventful. Within week the discharge had practically disappeared and in a month the wound had soundly granulated up from the bottom. A slight cough and a slight evening rise of tempera ture persisted for over two months. The patient was seen and examined in June, 1899, when she looked perfectly healthy and was free from cough. A slight bulging of the ribs and cartilages still persisted. The scar measured an inch in length and was situated in the fifth intercostal space. On auscultation the only adventitious sound heard in the chest was that of a dry rub in the immediate vicinity of the scar. Clydach, Swansea.

\section{A CASE OF PRIMARY ABSCESS OF THE KIDNEY.}

Bx J. STEWART, M.CH. R.U.I.

ON June 28th last a patient, aged 35 years, consulted me about a pain in her left side corresponding to the lower four or five ribs and for a slight cough. I found nothing abnormal in the chest with the exception of feebleness of breath sounds from the angle of the left scapula downwards. On July 1st I was asked to see her at home. The pain was still intense, but the cough was relieved and her temperature was $102^{\circ} \mathrm{F}$ : I examined the chest again and thought that I heard a pleuritic note at the base of the left lung. I visited her up to July 8th when she was so much improved that I ceased attending. On July 22nd she called to see me. The pain had returned, there were attacks of shivering, profuse night.sweats, and a temperature of $103^{\circ}$. On the day following I examined her in bed. With regard to the family history her father had died from phthisis at the age of 35 years and ber mother had died from epileptic mania; she had only one brother who died in infancy; she had no sisters. As to her personal history she had never had an illness before. The only thing noteworthy was her menstrual history; she menstruated once every six months, the first period being at the age of 18 years. In spite of this hitherto she had enjoyed most excellent health. A month before seeing me first-viz, on June 28th-she had just got rid of a cough, a cough unattended by pain anywhere and unaccompanied by expectoration. There was nothing but the cough, the patient not feeling ill during this time, but it was of such intensity that it made a most marked impression on the patient's mind, and was quite different from the slight cough which she had when I saw her. This intense cough lasted for a fortnight and suddenly ceased. I again examined the side, but this time also the abdomen, and 1 found a tumour in the left hypochondriac and lumbar regions. The tumour extended well into the loin, bulging it out slightly when viewed from above, the patient being in the recumbent position The tumour was smooth and felt solid; there was no fluctuation; it felt tender when pressed between the hands; it was rounded at its lower free end, but no free edge was detected either posteriorly or below such as one would find even in the largest spleen. It could be pushed well into the loin, filling it up; in front it extended to within one and a half inches of the mesial line and below to the level of the iliac crest. It had running down in front a line of partial resonance, and corresponding to this resonance a broad bard was felt, very tender on pressure, and which could be rolled underneath the fingers; it was no doubt the descending colon. The tumour moved up and down during respiration. Barwell looks upon this sign as distinguishing between a splenic and a renal tumour, the former moving up and down, the latter not. In this case it did distinctly travel. Notøithstanding this the tumour was clearly a renal one; it was also clear that it was associated with pus, judging from the pain, rigors, sweating, and temperature. Was it a hydro-nephrosis which bad become puralent-viz., a pyo-nepbrosis? It was to this opinion I leaned ; primary abscess never suggested itself, as the great size was against that view. Against the diagnosis of pyonephrosis there were strong arguments. There never had been an attack of renal colic or pain and there was nothing abnormal in the pelvic organs, such as tumours, \&c., causing pressure upon.the ureters. No albumin was present in the 